\title{
Robust Multi Sensor Pose Estimation for Medical Applications
}

\author{
Andreas Tobergte, Mihai Pomarlan, and Gerd Hirzinger
}

\begin{abstract}
In this paper a sensor fusion for pose estimation using optical and inertial data is presented. The proposed algorithm is based on extended Kalman filtering and fuses data from an optical tracking system and an inertial measurement unit. These two redundant sensor systems complement each other well, with the tracking providing absolute position accuracy and the inertial measurements giving low latency information of derivatives. Models for both sensors are given respecting the different sampling times and latencies. Another key issue is to use information about every landmark, i.e. marker, visible for the tracking system, by coupling the two sensor systems tightly together. The algorithms are evaluated in simulation and tested with an experimental hardware platform. The combined sensor system provides robust pose estimation in case of short time marker occlusion and effectively compensates for latencies the pose measurements.
\end{abstract}

\section{INTRODUCTION}

In modern operating rooms optical tracking systems are widely used for navigation. Especially in neurosurgery, surgeons navigate hand held tools equipped with reflecting marker balls while looking at a Monitor visualizing the tool in a virtual patient. Tracking systems are not only used in hand held navigated interventions but also in automated navigated control with robots. We developed an application for positioning of a biopsy needle with the Kinemedic, a versatile medical light-weight robot [12]. Fig. 1 shows the Kinemedic's successor, the MIRO with markers for optical tracking. In such applications tracking systems show three major drawbacks: (a) Occlusion of markers can lead to immediate loss of pose information. (b) Tracking systems are slow with low sampling rates and high latencies compared to a robots internal sensors. (c) Measurements are corrupted by noise, especially the orientation. A possible solution to this drawbacks is the additional use of an inertial measurement unit (IMU). Recent developments in Micro-ElectroMechanical Systems (MEMS) have lead to commercially availabe small size IMUs that give a three axis measurement of angular rates and translational acceleration. In combination with the tracking systems that is already standard in operation rooms an IMU could improve the quality of the measurements. In general the IMU data is very reliable, because it does not depend on external conditions, such as line of sight, and is insensitive to metal or magnetic fields. However, since only derivatives of position and orientation are measured, the numerical integration of IMU measurements will inevitably lead to a drift of computed poses. An

All authors are affiliated with the Institute of Robotics and Mechatronics, German Aerospace (DLR), 82234 Wessling, Germany andreas.tobergteddlr.de

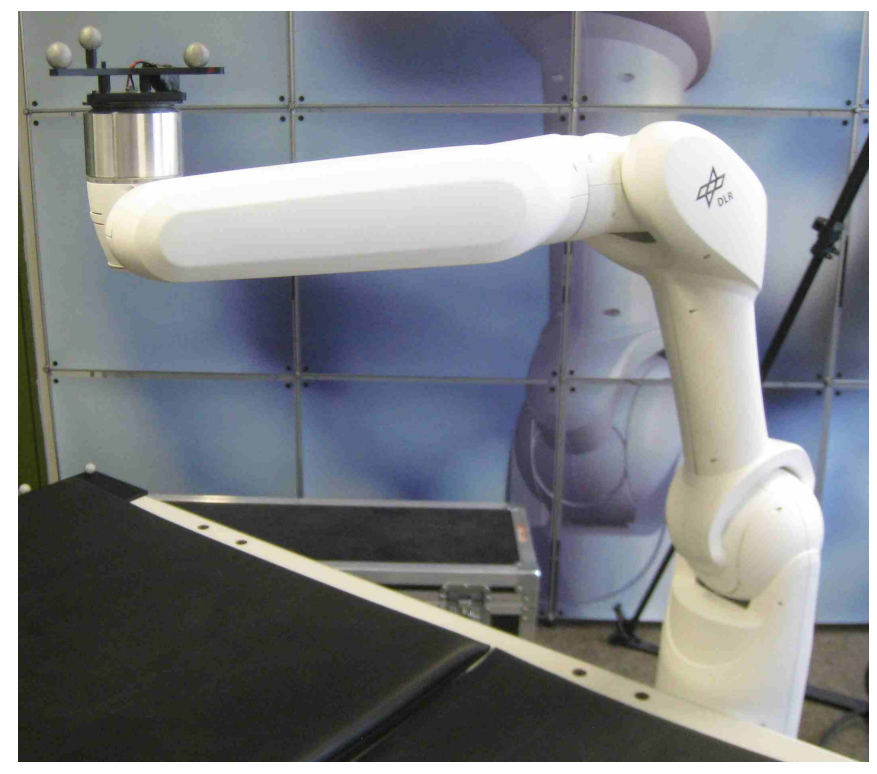

Fig. 1. The MIRO, a versatile medical light-weight robot, developed at the German Aerospace Center (DLR), with tracking markers of an optical tracking system attached to it

IMU is therefore not a substitute for a tracking system, but a suitable extension that costs a fraction of additional cameras and does not need much space in a crowded operation room. The quality of the combined data then depends on calibration, synchronization, sensor models and proper data fusion algorithms.

The authors identified three main goals for the combined system with optical tracking and IMU:

- The sensor fusion has to be able to estimate position and orientation in case of short time occlusion of tracking markers.

- Estimated position and orientation should not show significant delay.

- Orientation estimates should be reduced in noise.

- Combined sensor data should always be as good as or better than measurements of the tracking system.

The first, second and third goal aim to negate the most common performance drawbacks of optical tracking systems, as described above. The priorities of the goals depend on the specific application and can not be given in general. However, if the last goal is completely fullfilled the only reasons to use a tracking system without IMU would be either to reduce hardware effort or unfavorable environments for an IMU, e.g. the presence of radiation. However, the term 'as good or better' still needs to be defined. To achieve 
these goals the lower sampling rate and latency of the optical tracking system is rigorously modelled and the data fusion is performed with single marker position measurements instead of taking a 6-DoF pose measurement from the tracking system.

In section II a review of Kalman filtering and data fusion algorithms based on it is given. In the following section III the process and measurement models are derived based on sensor models. The data fusion is described and evaluated in simulation in section IV. A brief overview of the experimental hardware setup and results are given in section V. Section VI concludes the paper and gives an outlook on future work.

\section{STATE OF THE ART}

In this section a review of the Kalman filter and the extended Kalman filter is given. It is followed by a presentation of Kalman filter applications in navigation.

\section{A. Review of the Kalman Filter}

Since the publication of the Kalman filter [9], its variants have become the core of many sensor fusion applications. A detailed introduction is given in [6].

In the original formulation, the Kalman filter solves the problem of estimating the states of a linear system when noisy measurements of its outputs, and optionally measurements of its control inputs, are known. The Kalman filter is optimal, as there is no other filter which could better minimize estimation error covariance for linear systems in the presence of zero-mean mutually independent white gaussian noise. However, in practice most systems of interest are nonlinear, so an extended Kalman filter has been developed [5]. Let there be a system with the discrete state space model

$$
\begin{gathered}
\mathbf{x}[k]=\mathbf{f}(\mathbf{x}[k-1], \mathbf{u}[k], \mathbf{s}[k]) \\
\mathbf{y}[k]=\mathbf{g}(\mathbf{x}[k], \mathbf{r}[k])
\end{gathered}
$$

where as before $\mathbf{x}, \mathbf{u}$ and $\mathbf{y}$ represent state, control input and output vectors, $\mathbf{s}$ and $\mathbf{r}$ are process and measurement noise. The noise vectors at every step are mutually independent and are described by the covariance matrices $\mathbf{S}$ and $\mathbf{Q}$. State update and measurement functions are represented with $\mathbf{f}$ and g.

In that case, the filter first needs to calculate the matrices

$$
\begin{gathered}
\mathbf{A}=\left.\frac{\partial \mathbf{f}}{\partial \mathbf{x}}\right|_{\hat{\mathbf{x}}[k-1 \mid k-1], \mathbf{u}[k]} \mathbf{W}=\left.\frac{\partial \mathbf{f}}{\partial \mathbf{s}}\right|_{\hat{\mathbf{x}}[k-1 \mid k-1], \mathbf{u}[k]} \\
\mathbf{C}=\left.\frac{\partial \mathbf{g}}{\partial \mathbf{x}}\right|_{\hat{\mathbf{x}}[k \mid k-1]} \\
\mathbf{V}=\left.\frac{\partial \mathbf{g}}{\partial \mathbf{r}}\right|_{\hat{\mathbf{x}}[k \mid k-1]}
\end{gathered}
$$

where $[k \mid k-1]$ and $[k \mid k]$ were used to denote an a-priori, and respectively an a-posteriori, value calculated at time step $k$.
Once these Jacobians are obtained, the filter will proceed with the following formulas, for the predict

$$
\begin{gathered}
\hat{\mathbf{x}}[k \mid k-1]=\mathbf{f}(\hat{\mathbf{x}}[k-1 \mid k-1], \mathbf{u}[k], \mathbf{0}) \\
\mathbf{P}[k \mid k-1]=\mathbf{A} \mathbf{P}[k-1 \mid k-1] \mathbf{A}^{T}+\mathbf{W S W}^{T}
\end{gathered}
$$

and update phases

$$
\begin{aligned}
\mathbf{K}[k] & =\mathbf{P}[k \mid k-1] \mathbf{C}^{T}\left(\mathbf{C P}[k \mid k-1] \mathbf{C}^{T}+\mathbf{V} \mathbf{Q} \mathbf{V}^{T}\right)^{-1} \\
\hat{\mathbf{x}}[k \mid k] & =\hat{\mathbf{x}}[k \mid k-1]+\mathbf{K}[k](\mathbf{y}[k]-\mathbf{g}(\hat{\mathbf{x}}[k \mid k-1], \mathbf{0}))
\end{aligned}
$$

The error covariance matrix

$$
\mathbf{P}[k \mid k]=(\mathbf{I}-\mathbf{K}[k] \mathbf{C}) \mathbf{P}[k \mid k-1](\mathbf{I}-\mathbf{K}[k] \mathbf{C})^{T}+\mathbf{K}[k] \mathbf{Q K}^{T}[k]
$$

is given with the Joseph form [7], which guarantees that the covariance matrix is symmetrical and the computation is numerically stable.

The extended Kalman filter, or its further developed variants like the unscented Kalman filter [15], are generally not optimal estimators, due to the linearization. Furthermore, in many applications the assumption of zero-mean white Gaussian noise does not hold. Nevertheless, they have proven sufficient in practice, as their use in navigation shows.

\section{B. Kalman Filtering applied in Sensor data fusion}

In outdoor navigation the combination of IMU and GPS is widely used and algorithms for data fusion based of Kalman filtering are established, e.g. terrestrial vehicle navigation [14] [11]. Magnetic sensors, inertial angular rate, and gravity sensors were fused for motion tracking [8]. Terrain mapping is done with laser range finding, GPS and IMU data [10]. In augmented reality applications [4] [3] ultra sonic sensors, IMUs, and optical tracking are used.

In GPS/IMU systems, the concepts of loose, tight- and ultra-tight couplings have been developed, depending on the degree of processing done on satellite measurements before they are fed to the filter. As such, loose coupling means the filter is given the position calculated from time delay measurements of four satellites. Tight coupling means that the filter is given the time delay measurements of the visible satellites, and calculating the position is done inside the filter itself [11]. Ultra-tight coupling means using the EKF results to adjust the code tracking loop for the satellite signals [13], [2].

Another development in filter design is the Single Constraint at a Time, or SCAAT, technique, presented in [16], applied by e.g. [3]. It involves processing only one constraint, or measurement of the system output, when doing an update phase. The reason for doing this is, that acquiring a complete set of measurements takes more time than acquiring just one measurement, so in the interest of reducing latencies the measurements should be processed as soon as available. Observability is maintained because, after several update phases, a complete set of measurements is presented to the filter.

There were approaches of combining optical tracking and IMU data, e.g. in [1] a data fusion for medical applications 
based on optical tracking and inertial data is presented. It implements a loosly coupled data fusion using position and orientation measurements from the tracking system. Delays are modeled with time variant covariances. None of the approaches known to the authors consequently transferred advanced methods such as the tight coupling from outdoor to indoor navigation or models the differing behavior in time of the sensors explicitely.

\section{Modeling of The System}

Sensor data fusion requires proper models of the sensors. The optical tracking system and the inertial measurement unit are modelled with bias, latency, noise, and sampling times considered. Based on this IMU model a process model is derived, which basicly integrates the IMU measurements in space. The optical tracker provides data for the measurement model.

\section{A. Sensor models}

A measured sample of data coming from a sensor is defined as a set of a

- Value (scalar or vector) with a physical unit,

- Timestamp with the physical time corresponding to the value,

- Quality corresponding to the measured value, normalized in $[0,1]$.

The optical tracking system (OTS) provides a set of position measurements

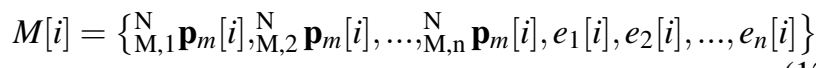

at a discrete step $i \in \mathbb{N}$ of $n \in \mathbb{N}(n \geq 3)$ marker balls and their corresponding quality $e_{j}$. Each marker measurement

$$
\mathbf{p}_{m, j}[i]={ }_{\mathrm{M}, \mathrm{j}}^{\mathrm{N}} \mathbf{p}\left(i \cdot t_{s, \text { OTS }}-t_{l, O T S}\right)+{ }_{\mathrm{M}, \mathrm{j}}^{\mathrm{N}} \mathbf{p}_{n}\left(i \cdot t_{s, \text { OTS }}-t_{l, O T S}\right)
$$

is given with the true position $\mathbf{p} \in \mathbb{R}^{3}$ at the physical time $\left(i \cdot t_{s, \text { OTS }}-t_{l, \text { OTS }}\right)$ with $t_{s, \text { OTS }}$ and $t_{l, \text { OTS }}$ being the sampling time and the latency respectively. Positions of marker points $\mathrm{M}, \mathrm{j} ; j \in\{1,2, \ldots, n\}$ and the noise $\mathbf{p}_{n}$ are given with respect to the navigation frame $\mathrm{N}$. The navigation frame is the frame of reference of the optical tracking system. It is fixed to the room and assumed to be an inertial frame.

The inertial measurement unit gives accelerations $\mathbf{a}_{m} \in \mathbb{R}^{3}$ and angular rates $\omega_{m} \in \mathbb{R}^{3}$ in 3 axis at the discrete step $k \in \mathbb{N}$ with the sampling rate $t_{S, I M U}$. The acceleration measured

$$
\begin{aligned}
\mathbf{a}_{m}[k] & ={ }_{\mathrm{S}}^{\mathrm{S}} \mathbf{a}\left(k \cdot t_{s, I M U}\right)+{ }_{\mathrm{S}}^{\mathrm{S}} \mathbf{a}_{b}\left(k \cdot t_{S, I M U}\right)+{ }_{\mathrm{S}}^{\mathrm{S}} \mathbf{a}_{n}\left(k \cdot t_{s, I M U}\right) \\
& +{ }_{\mathrm{S}}^{\mathrm{S}} \mathbf{a}_{g}\left(k \cdot t_{S, I M U}\right)
\end{aligned}
$$

is the true acceleration a corrupted with a bias $\mathbf{a}_{\mathbf{b}}$, noise $\mathbf{a}_{\mathbf{n}}$ and earth acceleration $\mathbf{a}_{g}$; all vectors are measured here in the sensor, or S, frame. Similarly, the measured angular rates

$$
\omega_{m}[k]={ }_{\mathrm{S}}^{\mathrm{S}} \omega\left(k \cdot t_{S, I M U}\right)+{ }_{\mathrm{S}}^{\mathrm{S}} \omega_{b}\left(k \cdot t_{s, I M U}\right)+{ }_{\mathrm{S}}^{\mathrm{S}} \omega_{n}\left(k \cdot t_{s, I M U}\right)
$$

are the true rates $\omega$ with bias $\omega_{\mathbf{b}}$ and noise $\omega_{\mathbf{n}}$. Unlike the OTS the IMU is assumed to measure without latencies. Furthermore the sampling time of the OTS is a multiple of the one of the IMU: $t_{s, \text { OTS }}=\psi \cdot t_{s, I M U}, \psi \in \mathbb{N}, \psi \geq 1$. Similarly, for the OTS latency $t_{l, \text { OTS }}=\rho \cdot t_{S, I M U}, \rho \in \mathbb{N}, \rho \geq 0$. Note that $i=0$ and $k=0$ correspond to the same physical time $t=0$.

\section{B. Process Model}

The task is to track an object as it moves in space. However, the IMU and the markers are rigidly fixed to this object. The tracking task can therefore be reduced to a tracking of the IMU with the markers attached to it.

The process model takes the inertial measurement as the control input vector

$$
\mathbf{u}[k]=\left(\mathbf{a}_{m}[k], \omega_{m}[k]\right)^{\mathrm{T}}
$$

and numerically integrates them to obtain the IMU motion with respect to the navigation frame. The state vector

$$
\mathbf{x}[k]=\left({ }_{\mathrm{S}}^{\mathrm{N}} \mathbf{p}[k], \stackrel{\mathrm{S}}{\mathrm{N}} \mathbf{q}[k], \underset{\mathrm{S}}{\mathrm{N}} \mathbf{v}[k], \stackrel{\mathrm{S}}{\mathrm{S}} \mathbf{a}_{b}[k], \stackrel{S}{\mathrm{~S}} \omega_{b}[k]\right)^{\mathrm{T}}
$$

is given with the position, the orientation quaternion and the velocity of the object in the navigation frame and the bias of the IMU. Based on the IMU model a state space model for the system with zero order hold discretization and system sampling time $t_{s}=t_{s, I M U}$ can be derived. The difference equations that determine the system matrix are:

$$
{ }_{\mathrm{S}}^{\mathrm{N}} \mathbf{p}[k]={ }_{\mathrm{S}}^{\mathrm{N}} \mathbf{p}[k-1]+t_{S \mathrm{~S}}^{\mathrm{N}} \mathbf{v}[k-1]
$$

$$
{ }_{\mathrm{S}}^{\mathrm{N}} \mathbf{q}[k]={ }_{\mathrm{S}}^{\mathrm{N}} \mathbf{q}[k-1] *\left[0, t_{S}\left(\omega_{m}[k]-{ }_{\mathrm{S}}^{\mathrm{S}} \omega_{b}[k-1]+{ }_{\mathrm{S}}^{\mathrm{S}} \omega_{n}[k]\right)\right]
$$

$$
\begin{aligned}
{ }_{\mathrm{S}}^{\mathrm{N}} \mathbf{v}[k]= & \underset{\mathrm{S}}{\mathrm{N}} \mathbf{v}[k-1]+t_{\mathrm{S}} \mathbf{R}\left({ }_{\mathrm{S}}^{\mathrm{N}} q[k-1]\right) \cdot \\
& \left(\mathbf{a}_{m}[k]-{ }_{\mathrm{S}}^{\mathrm{S}} \mathbf{a}_{b}[k-1]+{ }_{\mathrm{S}}^{\mathrm{S}} \mathbf{a}_{n}[k]\right) \\
& { }_{\mathrm{S}}^{\mathrm{S}} \mathbf{a}_{b}[k]={ }_{\mathrm{S}}^{\mathrm{S}} \mathbf{a}_{b}[k-1] \\
& { }_{\mathrm{S}}^{\mathrm{S}} \omega_{b}[k]={ }_{\mathrm{S}}^{\mathrm{S}} \omega_{b}[k-1]
\end{aligned}
$$$$
\left(\mathbf{a}_{m}[k]-{ }_{\mathrm{S}}^{\mathrm{S}} \mathbf{a}_{b}[k-1]+{ }_{\mathrm{S}}^{\mathrm{S}} \mathbf{a}_{n}[k]\right)-{ }_{\mathrm{S}}^{\mathrm{N}} \mathbf{a}_{g}
$$

$\mathbf{R}(\mathbf{q})$ is a rotation matrix equivalent to the orientation of a quaternion $\mathbf{q}$.

Then, the target objects's position, orientation and velocity in the $\mathrm{N}$-frame can be obtained, with ${ }_{\mathrm{O}}^{\mathrm{S}} \mathbf{p}$ and ${ }_{\mathrm{O}}^{\mathrm{S}} \mathbf{q}$ defining the transformation from the IMU to the object frame.

\section{Measurement Model}

For a single marker, the relationship between that marker's measured position in the $\mathrm{N}$-frame at a time $t=i \cdot t_{S, O T S}$, with latency $\rho \cdot t_{s, I M U}$, and the IMU's position and orientation in the $\mathrm{N}$-frame, at a time $(i \cdot \psi-\rho) \cdot t_{s, I M U}$ is given by

$$
\begin{aligned}
& \mathrm{M}, \mathrm{j} \mathbf{p}_{m}[i]={ }_{\mathrm{S}}^{\mathrm{N}} \mathbf{p}[i \cdot \psi-\rho]+\mathbf{R}\left({ }_{\mathrm{S}}^{\mathrm{N}} q[i \cdot \psi-\rho]\right)_{\mathrm{M}, \mathrm{j}}^{\mathrm{S}} \mathbf{p}_{m} \\
& +\quad \underset{\mathrm{M}, \mathrm{j}}{\mathrm{N}} \mathbf{p}_{n}[i \cdot \psi-\rho]
\end{aligned}
$$


where ${ }_{\mathrm{M}, \mathrm{j}}^{\mathrm{S}} \mathbf{p}_{m}$ is the position of the marker in the IMU frame and ${ }_{\mathrm{M}, \mathrm{j}}^{\mathrm{N}} \mathbf{p}_{n}[i \cdot \psi-\rho]$ is the measurement noise.

Whether a marker measurement ${ }_{\mathrm{M}, \mathrm{j}} \mathbf{p}_{m}[i]$ is used to build an observation model or not depends on whether the quality $e_{j}[i]$ of that measurement exceeds a predefined threshold $\theta$. The observation model will be built from a system of equations of the form shown in (23), with one equation for each marker with a sufficient quality.

\section{DATA FUSION}

The extended Kalman filter, as described in section II-A is applied to the models given in section III. Simulation results are plotted to verify the data fusion with the models given.

\section{A. Fusion model}

The state update $\mathbf{f}$ and measurement $\mathbf{g}$ functions are given with (18), (19), (20) and (23). Some differences to the straight-forward implementation arise, because of the latency of optical measurements, as well as their lower sampling rate than the inertial measurements. The filter runs a predict phase, whenever new inertial data is available, which means several successive prediction phases occur between update phases. During predict phases, the covariance matrix $\mathbf{P}$ increases to reflect the growing uncertainty of the estimations. State estimations, covariance matrices as well as inertial measurements are stored in a buffer, so that when an optical measurement with latency $\rho \cdot t_{s, I M U}$ becomes available at $t=i \cdot t_{s, O T S}=(i \cdot \psi) \cdot t_{s, I M U}$, the update step is applied to estimations obtained at an earlier time $(i \cdot \psi-\rho) \cdot t_{s, I M U}$. After an update phase, $\rho$ predict phases are run through the buffer, on the data stored within it, from the updated position until the present.

\section{B. Simulation}

The simulation generates a reference trajectory (position, orientation, velocity) with the states of the process model. Measured values for inertial quantities and markers were calculated from the true values using equations (14), (15) and (23), given a realistic noise level and bias. The process sampling time $t_{s}=t_{s, I M U}=2 \mathrm{~ms}$, while the optical system has $t_{s, \text { OTS }}=18 \mathrm{~ms}$ with latency $t_{l, \text { OTS }}=34 \mathrm{~ms}$. Three markers were simulated for tracking. Sinusoidal motion in 6-DoF of different magnitude and phase is simulated. The plots in Fig. 2 show the x-position (left column) and yaw-rotation (right column). In the first row, the system is simulated without occlusion of markers. The fused data (dashed line) follows the true value (solid line) tightly, whereas the measured position and orientation (dotted line) are delayed. In the second and third row, one and three marker were occluded (covered) respectively, from time $t_{o c}=15 \mathrm{~s}$ on. The pose measurements instantaneously get invalid with less than 3 visible markers. The position estimation tracks the reference with only two visible markers, whereas is drifts away, when no markers are visible anymore. The orientation shows no significant drift in this short time interval. This can also be seen in table I, where the corresponding root mean square (RMS) errors for the time interval $15 s-18 s$ are given. The
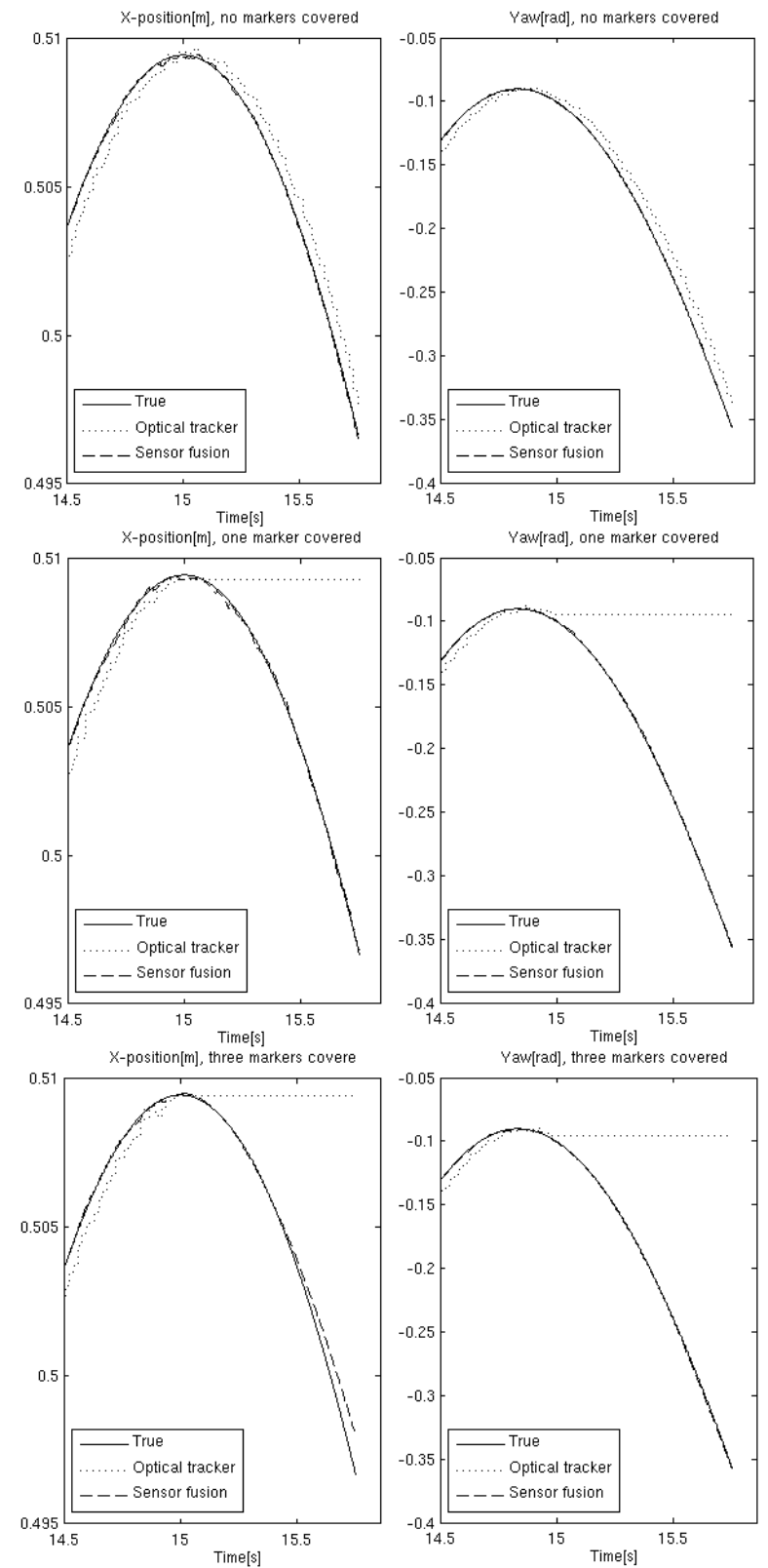

Fig. 2. Simulation plots with $\mathrm{x}$-axis (left) and yaw-angle (right) in the navigation frame. Three scenarios are shown: all markers visible (top), two markers visible (middle), all markers occluded (bottom). Marker occlusions occur at time $t_{o c}=15 \mathrm{~s}$

position estimates get worse the more markers are occluded with a huge step from one to no visible marker. The RMS errors for the optical tracker are given for a signal without delay to show that the estimation performs better even when working with a delayed pose measurement.

\section{EXPERIMENTS}

An experimental hardware setup was developed and experiments were performed in order to verify the simulation.

\section{A. Hardware setup}

The IMU used for experiments was an ADIS 16350 unit from Analog Devices, connected via a data acquisition card 
TABLE I

SIMULATION RESULTS SHOWING RMS ERRORS FOR A TIME INTERVAL OF 3 SECONDS WITH OCCLUDED MARKERS

\begin{tabular}{|l||l|l||l|l|}
\hline \multicolumn{1}{|c||}{} & \multicolumn{2}{c||}{ Optical tracker } & \multicolumn{2}{l|}{ Sensor fusion } \\
\hline $\begin{array}{l}\text { Markers } \\
\text { oc- } \\
\text { cluded }\end{array}$ & $\begin{array}{l}\text { Pos. X } \\
\text { RMSE } \\
(\mathrm{mm})\end{array}$ & $\begin{array}{l}\text { Yaw } \\
\text { RMSE } \\
\text { (rad) }\end{array}$ & $\begin{array}{l}\text { Pos. X } \\
\text { RMSE } \\
(\mathrm{mm})\end{array}$ & $\begin{array}{l}\text { Yaw } \\
\text { RMSE } \\
(\mathrm{rad})\end{array}$ \\
\hline 0 & 0.83 & 0.002 & 0.12 & 0.0006 \\
\hline 1 & - & - & 0.15 & 0.0011 \\
\hline 2 & - & - & 0.17 & 0.001 \\
\hline 3 & - & - & 29.2 & 0.0009 \\
\hline
\end{tabular}

to an off the shelf PC. The data fusion was processed on the computer running the QNX real-time operating system. Sampling time for the IMU was $t_{s, I M U}=2 m s$. Optical tracking was provided by the ART v2 system from Advanced Realtime Tracking Gmbh. It measures marker positions with a sampling time of $t_{s, O T S}=16 \mathrm{~ms}$ and a latency (estimated) of $t_{l, O T S}=34 \mathrm{~ms}$.

The experiments consisted of tracking the circuit board, where the IMU was mounted on. This circuit board was fixed on a support board, upon which three markers were aligned with the IMU x- and y-axis, see Fig. 3. Nevertheless, their position relative to the IMU is known with some error, and it remains as a future work to implement a procedure to calibrate marker position, relative to an IMU, accurately.

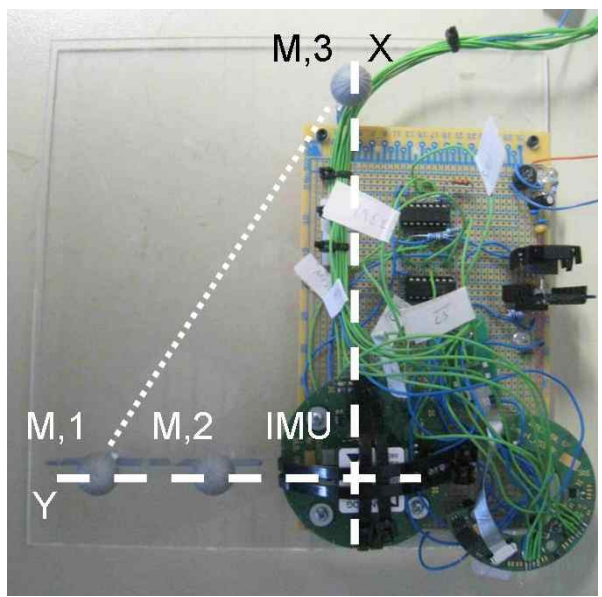

Fig. 3. The inertial measurement unit mounted on a circuit board with tracking markers $(\mathrm{M}, 1 ; \mathrm{M} 2 ; \mathrm{M} 3)$ aligned to the IMU's $\mathrm{x}$ - and $\mathrm{y}$-axis

\section{B. Experimental Results}

Like in the simulations, the state vector was estimated, in different marker occlusion scenarios. The IMU platform was moved in space manually in an unrestricted 6-DoF motion. The algorithms were processed four times on the same data, first with no marker occlusion, then with one, two and finally three markers occluded from time $t_{o c}=32.5 \mathrm{~s}$ on lasting for $3 s$. Markers were not physically occluded but instead invalidated in the software. This way the same set of data can be used for all cases. In Figure 4 the output of the sensor fusion algorithm (dashed line), is shown, versus the output obtained from the optical tracker (dotted line), as well as a reference (solid line). The reference trajectory was generated from the optical tracking data, that was smoothed and shifted forward by $t_{l, O T S}=34 \mathrm{~ms}$ to remove the latency. This reference enables an evaluation of performace in case of marker occlusions and latency but unfortunately not the performace concerning noise. The plots are grouped the same
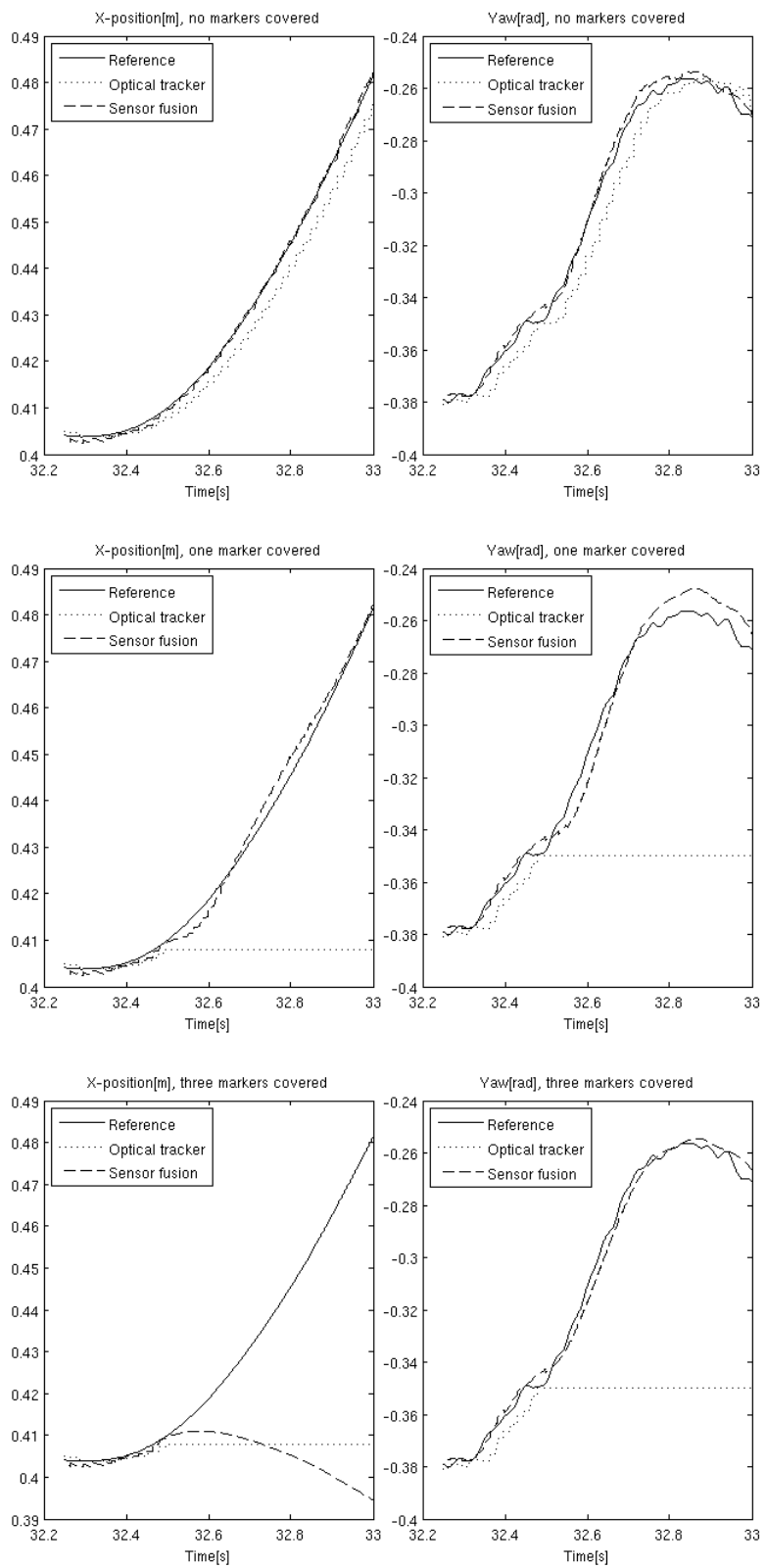

Fig. 4. Experimental plots with $x$-axis (left) and yaw-angle (right) in the navigation frame. Three scenarios are shown: all markers visible (top), two markers visible (middle), all markers occluded (bottom). Marker occlusions occur at time $t_{o c}=32.5 \mathrm{~s}$

way as for simulation and show similar results. The plots for the x-position and yaw-rotation in the first row show that the latency was effectively compensated. In the second row the marker $M, 2$ is occluded and ${ }_{\mathrm{M}, 2}^{\mathrm{N}} \mathbf{p}_{m}$ invalid, $e_{2}=0$ for 
TABLE II

EXPERIMENTAL RESULTS SHOWING RMS ERRORS FOR A TIME INTERVAL OF 3 SECONDS WITH OCCLUDED MARKERS

\begin{tabular}{|l||l|l|}
\hline \multicolumn{1}{|c||}{} & \multicolumn{2}{c|}{ Sensor fusion } \\
\hline $\begin{array}{l}\text { Markers } \\
\text { oc- } \\
\text { cluded }\end{array}$ & $\begin{array}{l}\text { Epos. } \\
\text { X RMS } \\
(\mathrm{mm})\end{array}$ & $\begin{array}{l}\text { Yaw RMS } \\
\text { (rad) }\end{array}$ \\
\hline 0 & 1.1 & 0.0028 \\
\hline 1 & 1.5 & 0.0078 \\
\hline 2 & 7.4 & 0.0157 \\
\hline 3 & 147.3 & 0.008 \\
\hline
\end{tabular}

$t_{s} \geq 32.5 \mathrm{~s}$. The rotation around the axis connecting M,1 and M,3 (dottet line in Fig. 3) is not measured and the position of the IMU ${ }_{S}^{N} \mathbf{p}$ can not be determined by the measurements, since it is not on this axis. Figure 4 show that the gyros can track the missing rotation for a few seconds and therefore also allow the observation of the IMU position. In the third row all markers are occluded and observability of all states is lost. The double integration of accelerometers, and the propagation of orientation errors to the position, leads to an instantaneous drift in the position, whereas the gyros can track the orientation for 3 seconds, as also shown in table II. When 6-DoF motion is performed, at least one marker has to be visible at any time for pose tracking. Another test was performed to determine the minimum requirements for long term pose estimation. It showed that the RMS error did not diverge in case of cyclic marker visibility. In every cycle first only $\mathrm{M}, 1$ was visible then only $\mathrm{M}, 2$, and finally only $\mathrm{M}, 3$, for $250 \mathrm{~ms}$ each. A stable pose estimation can be provided, if a least 1 marker is always visible and 3 markers are visible regularly.

\section{CONClusion AND Future Work}

The sensor fusion presented in this paper successfully combines an inertial measurement system with an optical tracking system. Models for both sensors are given and a state space model with process model and measurement model is derived. The sensor fusion couples the redundant sensors tightly using information about every visible marker for the estimation, while taking their different sampling rates and latencies into account. In case that only one marker is visible the orientation can be sufficiently estimated with the gyros for a few seconds and the pose is tracked with the marker position. If all marker are occluded the position estimation based on the accelerometers drifts away immediately. For good long time tracking performance, it is necessary that at least one marker is always visible and 3 different markers are visible regularly but not at the same time. The estimated pose tracks a reference trajectory in all cases without delay and a high resolution in time. Noise reduction is shown in simulation. The combined data can show errors in case of insufficient calibration or synchronization of the sensor systems which causes systematic errors the Kalman filter can not deal with optimally.
The presented methods will be integrated in applications, such as navigated robot control, registration or with a tracked input device for minimally invasive robotic surgery in the future. Calibration of IMU versus optical markers for generic marker geometries remains to be solved. A new IMU platform with integrated ethernet interface is currently designed. An interesting research topic will be the propagation of the quality of the measurements through the sensor data fusion to provide applications with a profound knowledge of the reliability of the current estimation.

\section{REFERENCES}

[1] Kotlarski J. Eilers H. Hofschulte J.-Heimann B. Baron, S. "Leistungsverbesserung eines optischen Tracking-Systems durch MultiSensordatenfusion". In Tagungsband der 6. Jahrestagung der Gesellschaft für Computer- und Roboterassistierte Chirurgie e.V., CURAC, pages pp. 279-282, 2007.

[2] Jinling Wang Di Li. Kalman filter design strategies for code tracking loop in ultra-tight gps/ins/pl integration. In Proceedings of the 2006 National Technical Meeting of the Institute of Navigation, pages 984 - 992, January 2006.

[3] George Pfeifer Eric Foxlin, Michael Harrington. Constellation: A wide-range wireless motion-tracking system for augmented reality and virtual set applications. In Proceedings of SIGGRAPH, pages 371-378, 1998.

[4] Eric Foxlin. Head-tracking relative to a moving vehicle or simulator platform using differential inertial sensors. In Proceedings of SPIE, volume Volume 4021: Helmet- and Head-Mounted Displays V, pages 133-144, April 2000.

[5] Leonard A. McGee Gerald L. Smith, Stanley F. Schmidt. Application of statistical filter theory to the optimal estimation of position and velocity on board a circumlunar mission. Technical Report R-135, NASA, 1962.

[6] Gary Bishop Greg Welch. An introduction to the kalman filter. SIGGRAPH 2001 Course 8.

[7] Matthew Barth Jay farrell. The Global Positioning System and Inertial Navigation. McGraw-Hill Professional, 1998.

[8] Eric R. Bachmann Robert B. McGhee Michael J. Zyda Joo Lus Marins, Xiaoping Yun. An extended kalman filter for quaternion-based orientation estimation using marg sensors. In Proceedings of the 2001 IEEE/RSJ International Conference on Intelligent Robots and Systems, pages 2003-2011, October-November 2001.

[9] Rudolf E. Kalman. A new approach to linear filtering and prediction problems. Research Institute for Advanced Study, Baltimore, Md., 1960.

[10] K. C. Slatton M. Sartori, M. Starek. Alsm data processing. Technical Report REP_2004-06-001, Geosensing Engineering and Mapping, Civil and Coastal Engineering Department of the University of Florida, June 2004.

[11] Salah Sukkarieh Michael George. Tightly coupled ins/gps with bias estimation for uav applications. In Proceedings of Australiasian Conference on Robotics and Automation (ACRA), December 2005.

[12] Tobias Ortmaier, Holger Weiß, Ulrich Hagn, Matthias Nickl, Alin Albu-Schäffer, Christian Ott, Stefan Jörg, Rainer Konietschke, Luc LeTien, and Gerd Hirzinger. A Hands-On-Robot for Accurate Placement of Pedicle Screws. In Proceedings of the 2006 IEEE International Conference on Robotics and Automation, 2006.

[13] Jinling Wang Ravindra Babu. Ultra-tight gps/ins/pl integration: Kalman filter performance analysis. GNSS 2005, Hong Kong, pages 8-10, 2005.

[14] Hugh F. Durrant-White Salah Sukkarieh, Eduardo M. Nebot. Achieving integrity in an ins/gps navigation loop for autonomous land vehicle applications. In Proceedings of IEEE Conference on Robotics and Automation, May 1998.

[15] Jeffrey K. Uhlmann Simon Julier. A new extension of the kalman filter to nonlinear systems. In International Symposium on Aerospace/Defense Sensing, Simulation and Controls, volume 3, 1997.

[16] Gregory Francis Welch. SCAAT: Incremental Tracking with Incomplete Information. $\mathrm{PhD}$ thesis, Department of Computer Science, University of North Carolina at Chapel Hill, October 1996. 